\title{
COMO INFLUYE LA DELINCUENCIA Y CORRUPCIÓN EN LAS ESTRATEGIAS DE GESTIÓN DE LOS DIRECTORES DE LAS MYPES
}

\author{
HOW CRIME AND CORRUPTION INFLUENCE THE MANAGEMENT \\ STRATEGIES OF MYPE DIRECTORS
}

\author{
Aracely Celina Sánchez-Albores ${ }^{1 *}$ (iD). \\ 1. Instituto Tecnológico Superior de la Región Sierra.
}

*Correspondencia del Autor: Aracely Celina Sánchez-Albores, correo electrónico: investigador_11@hotmail.com.

\section{RESUMEN}

Este artículo parte de la evaluación del impacto que tienen la delincuencia y corrupción en las estrategias gestión de las micro y pequeñas empresas del municipio de Teapa, Tabasco, la información se recolectó mediante un instrumento cuantitativo con una escala Likert de cinco puntos para efectuar el análisis sistémico y con preguntas dicotómicas, se realizó una investigación transversal y descriptiva, con una muestra de 465 mypes, se valoró el efecto de la delincuencia y corrupción en la toma de decisiones de los directores de las mypes del municipio citado. Se compararon las puntuaciones de cada variable del análisis sistémico en las empresas que han sido víctimas y en las que no lo han sido. Adicionalmente se analizó mediante regresiones lineales el efecto de la percepción de estas variables y como afecta a los negocios.

Palabras clave: Delincuencia; corrupción; estrategias de gestión; Mypes.

Cómo citar:

Sánchez-Albores, Aracely Celina. (2021). COMO INFLUYE LA DELINCUENCIA Y CORRUPCIÓN EN LAS ESTRATEGIAS DE GESTIÓN DE LOS DIRECTORES DE LAS MYPES. Revista de Investigaciones Universidad del Quindio, 33(1), 81-96. https://doi.org/10.33975/riuq.vol33n1.470 


\begin{abstract}
This article is part of the assessment of the impact of crime and corruption on the management strategies of micro and small enterprises in the municipality of Teapa, Tabasco, the information was collected through a quantitative instrument with a fivepoint Likert scale for systemic analysis and with dichotomous questions, a cross-cutting and descriptive investigation was carried out, with a sample of 465 mypes 5 mypes, the effect of crime and corruption on the decision-making of the directors of the mypes of the municipality cited was valued. The scores of each systemic analysis variable were compared in companies that have been victimized and have not been victimized. In addition, the effect of the perception of these variables and how it affects businesses were analyzed by linear regressions.
\end{abstract}

Keywords: Crime, corruption, management strategies, Mypes.

\section{INTRODUCCIÓN}

Las Micro y pequeñas empresas representan la base para el crecimiento económico de México, de acuerdo a la Secretaría de Economía (SE), del total de las empresas, a) el 95.2\% son Micro y pequeña empresas, b) generan el $45.6 \%$ del empleo y; c) aportan ingresos equivalentes al 23\% del Producto Interno Bruto (PIB) (Secretaría de Economía, 2019).

Funcionarios, políticos, empresarios y ciudadanos en general afirman que la delincuencia $y$ corrupción afecta las estrategias de gestión de las micro y pequeñas empresas del país convirtiéndose esto en un elemento inhibidor de la inversión, por ende de la creación de empleos y del crecimiento económico (Soria-Romo, 2017).

En los últimos doce años nuestro país ha sufrido una fuerte escalada en los rubros de delincuencia y corrupción de acuerdo a datos del (Foro Económico Mundial, 2018), en 2017 ubican a México en el lugar 135 de 180; con base en el índice de Percepción de la Corrupción 2017 (Transparency International, 2017), estos factores ha crecido progresivamente en la sociedad, causando gran preocupación por la inseguridad que la coloca en primer lugar con un $70 \%$.

Quiroz, Castillo, Ocegueda y Varela (2015) señalan que las personas ya no transitan por las calles con confianza, los empresarios temen invertir y la población en general vive en la incertidumbre de lo que le puede suceder ante los actos de violencia que cotidianamente presencian. A nivel individual, provoca cambios de conducta, en el intento de no sufrir un delito, que afectan al estilo y calidad de vida del ciudadano. A nivel colectivo, las repercusiones pueden ser muy destructivas para la vida comunitaria, en tanto se reduce la interacción social, se abandonan los espacios públicos o se rompe el control social informal (CIS,2000; Ruidíaz, 2003 \& Medina, 2003; Citado por Soto, 2005).

La violencia se relaciona negativamente con el desempeño económico en el largo plazo. Esto es, ambientes más violentos e inseguros se asocian con un desempeño menos favorable dela economía (Quiroz et al, 2015b). Así mismo, sugieren que la senda de crecimiento de la economía mexicana se ve significativamente afectada por el crimen, violencia e inseguridad reinantes en el país e indican que la variable de robos es la que cuenta con un número mayor de incidencia, llamando más la atención el incremento de las actividades delictivas que vulneran más significativamente la actividad económica: los homicidios y secuestros.

De acuerdo a Sansó,(2005) la delincuencia y la corrupción afronta respectivamente procesos evolutivos, adaptándose al entorno en el que pretende llevar a cabo sus actividades ilícitas, 
para evitar la pérdida de competitividad y eficacia, las nuevas amenazas se manifiestan cada vez con mayor dinamismo, si no se atajan tempranamente, su peligrosidad tiende a ir en un aumento vertiginosamente exponencial.

Coincidentemente con la importancia en lo que señala KPGM (2014) que dentro de las cinco variables para mejorar la competitividad en México están el combatir a la corrupción $80 \%$ y la seguridad Pública $79 \%$ y lo indicado en los estudios de Bernal y Castillo (2012) que muestran en su análisis cómo la violencia afecta la inversión extranjera directa en México y sus entidades federativas.

Es por ello, el (Instituto para la Economía \& Paz, 2017) menciona que la delincuencia tiene un costo que equivalente al $21 \%$ del PIB, es decir, 4.72 billones de pesos a los mexicanos, situando al 2017 como el año más violento de la historia.

Y en los últimos seis años, numerosos países han conseguido progresos mínimos o nulos". Esta afirmación es particularmente válida para Latinoamérica, como referencia podemos observar que, según la misma institución, en una escala de 0 a 100, donde 0 es considerado como más corrupción y 100 con menos corrupción, México esta evaluado con 29, Colombia y Perú con 37 y Ecuador con 32 (Transparency International Secretariat, 2018a).

Por consiguiente, se ubica a Tabasco en el lugar número de 24 en el tabulador "panorama del nivel de paz en México" con una calificación 3.161, entre el rango de estados "menos pacíficos", siendo los dos indicadores que inciden con mayor porcentaje son: Delitos cometidos con armas de fuego y Crímenes de la delincuencia organizada y corrupción (Índice de Paz México 2020a).

Cabe destacar, que los súbitos cambios económicos y sociales en Tabasco constituyen un elemento destacable en la ruptura de los límites sociales en la reducción de oportunidades que ocasionó la crisis petrolera, la cual propició que alrededor de 14000 tabasqueños pasaran a la franja de pobreza entre 2014 y 2016. La crisis propició también que el desempleo pasara de 7.3 a 8.2 entre 2017 y 2018, superando la media nacional de 3.2., y que el ingreso laboral per cápita real disminuyera $0.6 \%$ entre el cuarto trimestre de 2017 y el cuarto trimestre de 2018, al pasar de $\$ 1,364.73$ a $\$ 1,355.90$ pesos constantes, la cantidad menor desde 2014 (CONEVAL, 2018) citado por Ramírez (2019). En este sentido, la articulación entre el desempleo y la pobreza es un estímulo, entre muchos otros, que potencia la apertura de espacios para la acción social ilegal y la masificación de la delincuencia y corrupción.

En Tabasco se encuentran registradas 7,255 empresas de las cuales 6,150 son micro empresas $84.77 \%$ y pequeñas empresas $1,00813.90 \%$ (IIEG, 2018), sin embargo, han estado expuestas incremento de la delincuencia y corrupción, lo cual conlleva a replantear el comportamiento de los directivos de las mypes en la implementación de estrategias, derivado de la experiencia propia o por la percepción del riesgo.

En este sentido, se planteó el objetivo "El efecto de la delincuencia y la corrupción que ejerce en la toma de decisiones e implantación de estrategias de gestión de los directores de las mypes".

Seguidamente, se abordan resultados generales del trabajo de investigación entre ellos se encuentra la revisión de la literatura que sustenta este documento, se describe la metodología general utilizada en la investigación, de la misma manera se describen resultados de forma detallada comparado variable de análisis sistémico entre quienes han participado en actos de corrupción y quienes no y si el efecto de la delincuencia ha impactado en las personas, en lo económico y en el negocio, posteriormente se lleva a cabo la discusión que resulta importante analizar los resultados y comprar con otros autores el efecto de la delincuencia y corrupción. 


\section{REVISIÓN DE LA LITERATURA}

En este estudio, se presenta una descripción general de la situación de la delincuencia y corrupción en América Latina; más adelante, se describe acerca el contexto de las micro y pequeñas empresas (mypes) en la región. Con dicho contexto se discutirá el efecto que la delincuencia y la corrupción que ejerce en la toma de decisiones de los directores de las mypes, es decir, en las estrategias de gestión,

En el presente trabajo abordamos dos variables la delincuencia y la corrupción, que pueden afectar las estrategias de gestión de los directores de mypes : por un lado, abordamos la victimización por parte de la delincuencia, entendida como haber sido víctima del "grupo de delitos que las personas perciben como frecuentes $\mathrm{o}$, alternativamente, graves, y de los cuales pudieran llegar a ser víctimas ellos personalmente o sus familiares o conocidos" (Mertz, 2012, p. 2); por otro lado, abordamos la valoración subjetiva de la experiencia de victimización.

Diversos autores coinciden en que Latinoamérica es una de las regiones en donde se registra mayor presencia de delincuencia en el mundo (BriceñoLeón \& Zubillaga, 2002a; Soares \& Naritomi, 2010a).Por lo regular se utiliza el número de homicidios por cada 100,000 habitantes como un índice de prevalencia de la delincuencia y de la violencia en un país o una zona determinados. En la tabla 1 se presentan dichos índices para los países en donde se realizó el estudio.

Tabla 1.

Tabla 1. Índice de asesinatos por 100,000 habitantes.

\begin{tabular}{|l|l|l|l|}
\hline \multicolumn{1}{|c|}{ País } & Año 1998 & Año 2008 & Año 2017 \\
\hline Argentina & & & 5.1 \\
\hline Colombia & 58.8 & 35.9 & 24.6 \\
\hline Cuba & & & \\
\hline Ecuador & 13.5 & 18 & \\
\hline México & 13.8 & 12.3 & 24.8 \\
\hline Perú & & & 7.7 \\
\hline \multicolumn{4}{|c|}{ Datos tomados de UNODC (2019). } \\
\hline
\end{tabular}

Datos tomados de UNODC (2019).
Para dimensionar el problema, consideremos que, de acuerdo con la encuesta Internacional de Víctimas del Crimen, alrededor de 44\% de latinoamericanos son víctimas de algún crimen cada año (Soares \& Naritomi, 2010Bb). Según Jaitman y Keefer (2017), con base en el número de homicidios de la región, $33 \%$ de los homicidios perpetrados en el mundo en 2015 sucedió en América Latina, en donde sólo vive 9\% de la población mundial; esto habla de que no sólo se tiene una alta incidencia de la delincuencia, sino que además es una región muy violenta.

Se ha encontrado que el crimen suele florecer cuando los potenciales delincuentes consideran que las ventajas de delinquir son más grandes que el riesgo de ser arrestado o encarcelado (Motta, 2017a). En esa línea de pensamiento, Soares y Naritomi (2010c) consideran que la delincuencia en la zona se puede explicar en gran medida por tres factores: la desigualdad social, la baja tasa de encarcelamientos y el reducido tamaño de las fuerzas policiacas; los tres factores magnifican el principio presentado por Motta (2017b), ya que la diferencia de distribución de recursos hace que se vean atractivas las posibles ventajas del delito, mientras que la baja tasa de encarcelamientos y el tamaño de las fuerzas policiacas se ven como una circunstancia de bajo riesgo.

Por otro lado, algunos autores mencionan que hay evidencias contradictorias respecto al efecto que tiene la presencia de los cárteles en los distintos países, ya sea como proveedores, como rutas de paso o como productores, ya que hay casos en los que el incremento o disminución de las tasas de criminalidad no están acompañadas de diferencias atribuibles a las actividades de estas organizaciones (Soares \& Naritomi, 2010d). Quizá esto se deba a que la delincuencia requiere de factores endógenos a las sociedades en donde se gesta (bajo riesgo de ser castigado) y que los cambios se deban más a este factor que a las oportunidades (como el colaborar con el narcotráfico).

El miedo a ser víctima de la delincuencia suele 
llevar a las personas a tomar decisiones que afectan su estilo de vida (Briceño-León \& Zubillaga, 2002b), los efectos de la delincuencia y la violencia más frecuentemente estudiados se han centrado en resultados socioeconómicos como la reducción de la esperanza de vida, la incidencia en la calidad de vida y el costo que tienen dichos efectos en la economía; sin embargo, se ha estudiado en menor medida sobre los cambios de comportamiento de la población a raíz del fenómeno.

El hecho de que las personas ajusten sus patrones de vida es otro modo en que la delincuencia afecta a una sociedad y puede tener también consecuencias económicas. Por ejemplo, se sabe que en los lugares en donde la delincuencia es elevada, las personas disminuyen el tiempo que están en la calle (Soares \& Naritomi, 2010e), esto en sí mismo afecta la actividad económica y como consecuencia también las finanzas de las mypes; además, da pie para pensar que puede haber otros cambios de comportamiento relacionados con formas de consumo, y del lado del empresario, en la forma en la que deciden gestionar la empresa. Otro fenómeno social provocado por la delincuencia, como lo comentan Corbacho, Philipp y Ruiz-Vega (2015a), es la falta de confianza entre las personas que puede promover bajos deseos de cooperación, favorecer la aparición de instituciones y en última instancia empresas débiles, con una reducción de oportunidades de negocio y de crecimiento.

Algunos autores señalan que la pobreza, medida por ejemplo mediante el Producto Interno Bruto (PIB) per cápita de un país, tiene un efecto en la presencia de la delincuencia (Islam, 2014). Si bien la región es significativamente más pobre y registra menos años de educación formal que otras regiones del mundo, no hay un consenso en el hecho de que exista un vínculo inequívoco entre estas dos variables y la delincuencia (Soares \& Naritomi, 2010f), por lo que es equivocado afirmar categóricamente que la delincuencia se da en los lugares donde hay pobreza. Es más correcto pensar que la pobreza se da en lugares donde hay mucha desigualdad económica, ya que es una circunstancia en la que la delincuencia se presenta como una oportunidad de movilidad social (Lora, 2008a).

Una consideración relevante es la diferencia entre la delincuencia real de una región y la percepción de inseguridad que sienten los ciudadanos derivada de ésta, pues es la percepción la que lleva a los ciudadanos a cambiar sus patrones de vida. Por ejemplo, según Dammert y Malone (2006), el apoyo a la democracia por parte de la ciudadanía, las preferencias electorales o su participación en manifestaciones públicas, se debe a la percepción subjetiva de la delincuencia. Los mismos autores explican que el miedo a la delincuencia es producto de diversos factores como la cobertura de los medios, la inseguridad económica y política, la confianza en la aplicación de la ley, entre otros factores.

En el mismo sentido, Lora (2008b) menciona que la percepción de la delincuencia está más influenciada por los cambios en los niveles reales de delincuencia que en el nivel por sí mismo, infiriéndolo del hecho de que en países como Colombia la gente está más satisfecha que en Uruguay con los niveles de delincuencia, ya que en el primero, teniendo un índice de homicidios mucho más alto que el de Uruguay, éste ha bajado considerablemente, mientras que en Uruguay ha aumentado.

De igual importancia, diferentes autores coinciden que la corrupción es uno de los cánceres que más daño hacen a las mypes en Latinoamérica y el efecto que tiene en la toma de decisiones de los directores o, dicho de otro modo, si su enfoque o estrategia se modifica por la presencia de esta. La corrupción es un fenómeno difícil de definir y operacionalizar (Transparencia Internacional, 2017), la corrupción se define como "el uso de un poder público para fines privados", ésta es poco operable y excluye elementos importantes de su definición (Del Castillo, 2003).

Como ha señalado Friedrich (2002), existen 
diversas connotaciones del concepto de corrupción en todos los idiomas, pero, además, las definiciones son distintas dependiendo de la perspectiva que se adopte. Así, pueden existir importantes diferencias en lo que se entiende por corrupción según la definan las leyes o la opinión pública o las autoridades (Gardiner, 2002).

De acuerdo con Mendoza, Lim y Lopez (2015), a pesar del interés reciente por investigar los efectos de la corrupción en la empresa, los estudios han sido limitados y sus resultados no han sido conclusivos en cuanto al grado en el que la corrupción ayuda o afecta a la organización. Entre los efectos estudiados se ha encontrado que, a niveles más altos de corrupción, se encuentran tasas más bajas de productividad y de menor crecimiento de la producción.

Las razones encontradas para que los empresarios recurran a actos de corrupción se pueden englobar en dos categorías: a) para compensar el fracaso del sistema legal en la obtención de permisos o eludir procesos y trámites, mediante el pago de sobornos (Aidis \& Adachi, 2007), o b) para obtener alguna ventaja frente a la competencia (Williams \& Vorley, 2015).La corrupción, a nivel macroeconómico, tiene diversos efectos negativos en la sociedad, tales como la reducción del crecimiento económico de los países (Baumol, 1990; Boudreaux, Nikolaev \& Holcombe, 2018; Dimant \& Tosato, 2018; Morlai, 2015).

De manera paradójica, los efectos de la corrupción no siempre afectan a la sociedad; por ejemplo, se encontró que la presencia de corrupción en la sociedad no afecta el espíritu empresarial e incluso en los países con los peores climas de negocios se tienen altas tasas de emprendimiento (Álvarez \& Urbano, 2011; Dutta \& Sobel, 2016). Observando los índices de corrupción de los países divulgados por Transparency International Secretariat (2018b: párr. 5), encontramos "un dato perturbador: a pesar de los esfuerzos por combatir la corrupción en el mundo, la mayoría de los países están avanzando con demasiada lentitud.
En México, la corrupción es un hecho endémico que afecta enormemente tanto lo público como lo privado. En el primer caso, muchos estudios han mostrado que el soborno o lo que popularmente se conoce como "mordida" es el acto que la mayoría de los mexicanos identifica con corrupción. Además, su práctica es asociada principalmente con la Policía cuyos integrantes son percibidos como altamente corruptos y muy vinculados con el crimen (Del Castillo \& Guerrero, 2003; Bailey \& Parás, 2006; Naval \& Salgado, 2006), más sin embargo, en México la corrupción se ha convertido como parte de las reglas informales que manejan las interacciones entre policías y ciudadanos (Sabet, 2013).

\section{Contexto mype}

De hecho, la delincuencia es considerada por los micro empresarios como el problema principal para el crecimiento de las micro y pequeñas empresas, incluso por encima de la corrupción, y este fenómeno se mantiene tanto en países desarrollados como en los no desarrollados ( Mthimkhulu \& Aziakpono, 2018).

La Encuesta Nacional de Victimización de Empresas (ENVE, 2018) señala la prevalencia delictiva sobre la seguridad pública y desempeño de las autoridades en las unidades económicas empresariales durante el 2017, el $38.40 \%$ se dio en los comercios fueron víctimas del delito, $30.60 \%$ del sector Industria y $29.3 \%$ del sector servicio y de estas el $59.30 \%$ son empresas grandes, $61.50 \%$ medianas, $51.40 \%$ pequeñas $\mathrm{y}$ $32.90 \%$ micro.

Por otra parte, en el análisis de la Encuesta Nacional de Victimización de Empresas (ENVE, 2016) explica que $57,4 \%$ de las empresas encuestadas considera la inseguridad como el problema que más les afecta. De acuerdo con los datos reportados por procuradurías y fiscalías estatales al Secretariado Ejecutivo del Sistema Nacional de Seguridad Pública (SESNSP), entre los meses de enero y agosto de 2017 el robo a negocio superó su record histórico: se iniciaron 
61720 carpetas de investigación por este delito, $43 \%$ cometidos con violencia. El número de carpetas de investigación registradas por robo a negocio aumentó $37,6 \%$ frente al mismo período de 2016 y $14 \%$ frente al total del año 2011, cuando se reportó el pico estadístico. Las entidades federativas con mayor número de carpetas de investigación por este delito fueron Ciudad de México (19\%), Baja california (9.20\%), Estado de México (8.7\%), Tabasco (6.8\%) y Guanajuato (6.2\%) (Rodríguez, 2017).

Después de todo, es importante conocer cada tipo de victimario para establecer acciones de política tendientes a enfrentar la comisión de delitos contra las empresas. Como se aprecia en el siguiente esquema 1 en donde se describe diferentes tipos de victimarios de empresas y los principales delitos que cometen contra las unidades de económicas.

Esquema 1. Delitos contra las empresas por tipo de victimario más probable

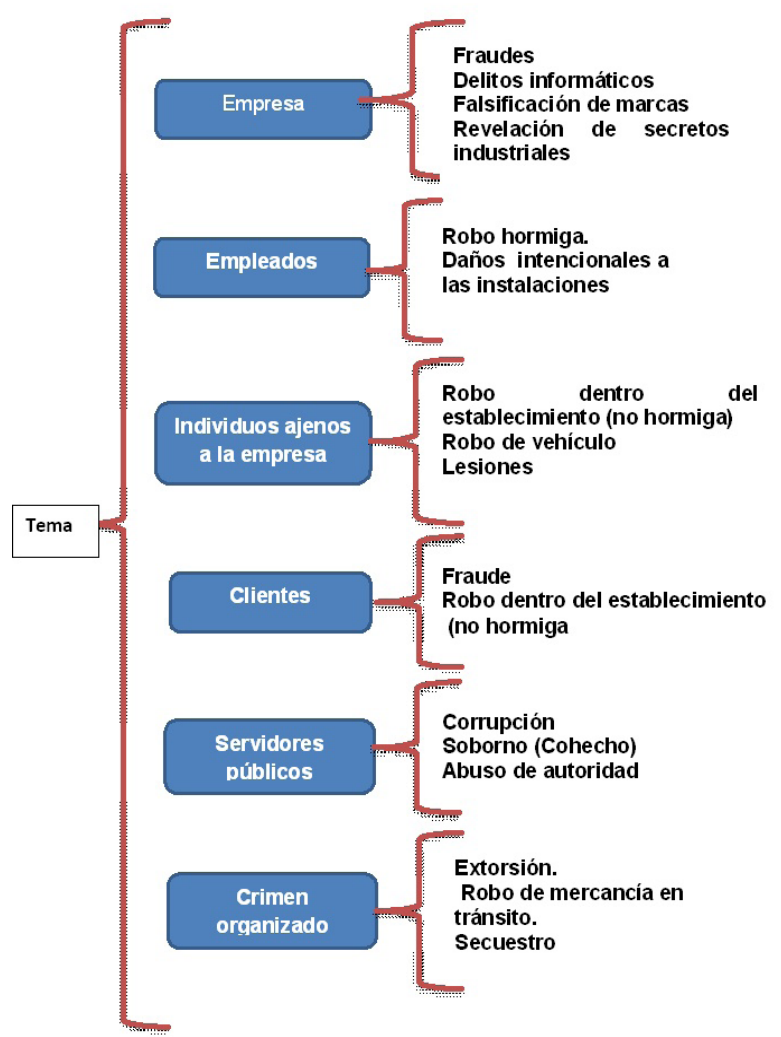

Fuente: elaboración propia con base en Vilalta (2016).
Efectos de la delincuencia y corrupción en la toma de decisiones de las mypes.

Por otro lado, la intensificación de la delincuencia en México aumenta cada día y los riesgos para los trabajadores que diariamente se trasladarse al centro de trabajo, repercutiendo esto en la disponibilidad de los empleados para laborar horas extras, ya que estar en la calle a ciertas horas se torna peligroso, datos estadísticos del mes de marzo del 2021 la percepción de inseguridad es de $78.40 \%$ de la población de 18 años y más señalo sentirse insegura en cajeros automáticos localizado en la vía pública, mientras que el $71.20 \%$ se sintió insegura en el transporte público, calles que habitualmente usan $61.30 \%$, carretera $53.40 \%$, automóvil $35.60 \%$ y en el trabajo $34.40 \%$ (INEGI,2021).

Sin embargo, "es interesante señalar que las medidas objetivas de la delincuencia no siempre se correlacionan con las percepciones" (Lora, 2008c, p. 224), por otra parte, la corrupción es un fenómeno complejo con múltiples causas y efectos, que fluctúa desde el simple acto de un pago ilícito hasta el funcionamiento endémico del sistema económico y político (Del Castillo, 2003).

Las empresas latinoamericanas consultadas experimentan dificultades para admitir como un riesgo la existencia de actos de corrupción. Pese a los estudios que reportan una incidencia creciente de estos delitos, los gerentes entrevistados mostraron reservas para admitir que la corrupción puede existir en sus propias empresas, 68\% respondió que la corrupción es un fenómeno de escasa incidencia, 5\% contestó que existía algún nivel de incidencia en sus negocios. Sin embargo, el 53\% de los ejecutivos consultados respondió que la corrupción es un tema muy importante que requiere ser discutido y atendido, de igual forma el $45 \%$ considera poco probable que su empresa enfrente casos de corrupción en el futuro, $22 \%$ casi probable la expectativa de que 
su empresa enfrente este tipo de delitos en el futuro (KPMC,2019).

\section{MÉTODO}

El diseño de la investigación es transversal, la recolección de datos se realizó en un solo momento; también es descriptivo y tiene como objetivo indagar el nivel de una o más variables en una población; en el caso de este estudio, es conocer el grado de delincuencia percibida por los directores de las micro y pequeñas empresas del municipio de Teapa, Tabasco, a partir del análisis sistémico, y así medir su efecto en sus estrategias de gestión.

Para ponderar el efecto de la delincuencia y la corrupción en las estrategias de gestión, se harán dos análisis. Por un lado, se compararán las medias de los niveles de cada elemento del sistema presentados en la tabla, determinando si las empresas que fueron víctimas de la delincuencia y corrupción y presentan niveles diferentes de las empresas que no fueron víctimas. Por otro lado, se hace una regresión lineal para cada elemento del sistema en el que se valora el efecto de la percepción de delincuencia y corrupción, haber sido víctimas de la delincuencia-corrupción en los distintos componentes de la estrategia.

\section{Contexto local}

La investigación fue realizada en el municipio de Teapa, Tabasco, el cual tiene una población de 58523 habitantes; la edad promedio de la población en general es de 26 años; por cada 100 personas en edad productiva hay 52.3 en edad de dependencia económica; el promedio de habitantes por vivienda es de 3.8 personas, $10.1 \%$ de la población no cuenta con instrucción educativa, 59\% tiene educación básica, 18.3\% media superior y $12.4 \%$ superior. La población económicamente activa (PEA) alcanza 48.7\% y la no activa 51.3\% (Instituto Nacional de Estadística y Geografía, 2015). El municipio cuenta con 1983 unidades económicas, de las cuales 1959 son micro y pequeñas empresas, que representan 98.8\% (DENUE, 2019).

\section{Muestra}

La población a estudiar está conformada por los directores de las micro y pequeñas empresas del municipio de Teapa, Tabasco. Para efectos de esta investigación, el director o directora de la mype es la persona que toma la mayoría de las decisiones en la organización y una mype es cualquier organización con fines de lucro que tiene al menos a una persona trabajando para un patrón y que cuenta máximo con 50 personas en la organización. El tamaño de la muestra fue de 465 directores y directoras de mype, lo que permite tener un nivel de significancia de 5\%. La recopilación de la información se llevó a cabo con participación de 50 alumnos del nivel superior previamente capacitados, tanto en la aplicación del cuestionario como en la captura de los mismos en la plataforma correspondiente.

Además, 42.6\% de los directores de la muestra es mujer, con una edad promedio de 41.6 años; el estado civil más representado es casado, con $54.2 \%$. Respecto a la escolaridad, se encontró que 9.7\% de los directores no cuenta con educación formal, $10.1 \%$ estudió primaria, $27.7 \%$ estudió secundaria, $34.6 \%$ estudió bachillerato, $16.8 \%$ terminó la licenciatura y el restante $1.1 \%$ tiene estudios de posgrado. Se estima que, en promedio, los directores dedican 8.78 horas a la semana a su negocio. En la tabla 1 se presentan las actividades a las que se dedican las empresas participantes en el estudio. 
Tabla 1. Actividad de las micro y pequeñas empresas del municipio de Teapa.

\begin{tabular}{|l|c|}
\hline \multicolumn{1}{|c|}{ Actividad } & $\mathbf{\%}$ \\
\hline Agricultura, ganadería, silvicultura y pesca & 1.290 \\
\hline Explotación de minas y canteras & 0.000 \\
\hline Industrias manufactureras & 12.280 \\
\hline $\begin{array}{l}\text { Suministro de electricidad, gas, vapor, aire acondicionado y agua; evacuación de aguas } \\
\text { residuales, gestión de desechos y descontaminación }\end{array}$ & 0.000 \\
\hline Construcción & 0.430 \\
\hline Comercio al mayoreo y reparación de vehículos y motocicletas & 11.210 \\
\hline Comercio al menudeo & 43.100 \\
\hline Transporte y almacenamiento & 0.650 \\
\hline Actividades de alojamiento y servicio de comidas & 16.810 \\
\hline Información y comunicaciones & 3.230 \\
\hline Actividades financieras y de seguros & 0.000 \\
\hline Actividades inmobiliarias & 0.000 \\
\hline Actividades profesionales, científicas y técnicas & 1.290 \\
\hline Actividades de servicios administrativos y de apoyo & 1.290 \\
\hline Actividades de enseñanza & 0.220 \\
\hline Actividades de atención de la salud humana y de asistencia social & 1.720 \\
\hline Actividades artísticas, de entretenimiento y recreativas & 1.940 \\
\hline Otras actividades de servicios & 3.880 \\
\hline $\begin{array}{l}\text { Actividades de los hogares como empleadores; actividades no diferenciadas de los } \\
\text { hogares como productores de bienes y servicios para uso propio }\end{array}$ & 0.65 \\
\hline
\end{tabular}
Fuente: elaboración propia con base en los resultados de la investigación 2020.

\section{Instrumento de investigación}

El cuestionario fué diseñado para ser contestado en papel por el director o directora de la empresa, dando la opción a que los encuestadores pudieran leerlos y rellenarlos de acuerdo con la información proporcionada por la persona entrevistada.

Las variables de la investigación están contenidas en varias secciones del cuestionario aplicado a los participantes. Las estrategias de gestión fueron evaluadas por 105 ítems tipo Likert de 5 niveles (muy de acuerdo, de acuerdo, en desacuerdo, muy en desacuerdo, nosé/no aplica) que conforman el análisis sistémico, dividido en insumos del sistema [proveedores (6 ítems), análisis de mercado (7 ítems) y recursos humanos (12 ítems)], procesos del sistema [dirección (8 ítems), gestión de ventas (6 ítems), innovación
(8 ítems), producción-operación (5 ítems), mercadotecnia (9 ítems) y finanzas (13 ítems)], resultados del sistema [satisfacción con la empresa (5 ítems), ventaja competitiva (6 ítems), ámbito de ventas (6 ítems), principios ISO 26000 (8 ítems, uno por cada principio), asuntos ISO 26000 (6 ítems, uno por cada asunto), las últimas dos variables se encuadran en una sola, llamada Responsabilidad social ISO 26000. Para todas las estrategias evaluadas se consideró el promedio aritmético de las respuestas de la variable, salvo para el ámbito de ventas, al que se le aplicó un promedio ponderado dándole el doble de peso a quien tiene ventas de importancia en la ciudad, el triple a quien tiene ventas en otras poblaciones, a otras empresas o al gobierno, y el cuádruple a quien tiene ventas en el extranjero.

Para medir la experiencia de la delincuencia y 
corrupción se utilizó una lista de nueve delitos que afectan a las empresas, ante los que los empresarios contestaban cuántas veces habían sido víctimas de ese delito; para efectos del estudio, se clasificaron únicamente en dos categorías: quienes habían experimentado algún delito $\mathrm{y}$ quienes no lo habían vivido, ni ellos directamente ni alguien de la empresa. La percepción del delito se midió en dos niveles, por un lado, se les preguntó con ítems dicotómicos (sí/no) si existían diversos fenómenos de delincuencia en los alrededores, tales como robo de mercancía, secuestros, homicidios, extorsión, por ejemplo, y, por otro lado, se les preguntó si creían que esos fenómenos afectaban a la empresa (10 ítems en cada nivel).

\section{Estadística de las variables y confiabilidad}

A continuación, se presenta la estadística descriptiva de las variables de estudio en la muestra de 465 mypes. Los datos fueron analizados en el programa R versión 3.6.1., usando el paquete psy versión 1.1 para el alfa de Cronbach.

En la tabla 2 se presentan las medias de las escalas de las variables del análisis sistémico de la mype, así como su alfa de Cronbach por escala. Se puede observar que sólo la variable de satisfacción con la empresa tuvo un alfa de Cronbach menor a 0.75 .

Tabla 2. Estadística descriptiva de la muestra.

\begin{tabular}{|l|c|c|c|}
\hline \multicolumn{1}{|c|}{ Variable } & Media & $\begin{array}{c}\text { Desviación } \\
\text { standar }\end{array}$ & $\begin{array}{c}\text { Alfa de } \\
\text { Cronbach }\end{array}$ \\
\hline Recursos humanos & 3.859 & 1.051 & 0.963 \\
\hline Análisis de mercado & 3.832 & 0.778 & 0.874 \\
\hline Proveedores & 4.112 & 0.818 & 0.886 \\
\hline Dirección & 3.780 & 0.721 & 0.816 \\
\hline Finanzas & 3.803 & 0.758 & 0.881 \\
\hline Gestión de ventas & 3.479 & 0.972 & 0.865 \\
\hline Producción - Operación & 4.351 & 0.679 & 0.875 \\
\hline Innovación & 3.194 & 1.003 & 0.893 \\
\hline Mercadotecnia & 3.632 & 0.748 & 0.838 \\
\hline Satisfacción con la empresa & 3.847 & 0.614 & 0.668 \\
\hline Ventaja competitiva & 4.149 & 0.731 & 0.882 \\
\hline Ámbito de ventas & 3.178 & 1.014 & 0.804 \\
\hline Responsabilidad social ISO & 3.773 & 1.022 & 0.954 \\
\hline Valoración del entorno & 3.972 & 0.721 & 0.894 \\
\hline
\end{tabular}

Fuente: elaboración propia con base en los resultados de la investigación 2020.

Sobre las variables de delincuencia, se encontró que $83.7 \%$ mencionó que, según su percepción, al menos 1 de los 10 delitos enlistados estaba presente en los alrededores de la empresa, mientras que $45.4 \%$ mencionó que consideraba que esos delitos afectaban a su empresa. Por otro lado, 25.9\% de los participantes de la zona estudiada mencionó haber sido víctima de la delincuencia en el último año.
En promedio, aquellos que fueron víctimas mencionaron haber sido víctimas de 5.3 delitos durante el año, en cuanto a la corrupción el 3\% de ellos consideran que en los últimos tres años la corrupción que debe soportar la empresa ha seguido igual, de igual manera se presenta en la tabla 3 la descripción estadística de las variables de confiabilidad en relación a la corrupción. 
Tabla 3. Estadística de las variables de confiabilidad.

\begin{tabular}{|l|c|c|c|c|}
\hline \multicolumn{1}{|c|}{ Variable } & Media & $\begin{array}{c}\text { Desviación } \\
\text { estándar }\end{array}$ & $\begin{array}{c}\text { Alfa de } \\
\text { Cronbach }\end{array}$ & $\begin{array}{c}\text { Correlación } \\
\text { con la } \\
\text { corrupción }\end{array}$ \\
\hline Proveedores & 4.111957 & 0.8181046 & 0.8856542 & 0.05488 \\
\hline Análisis de mercado & 3.832234 & 0.7782270 & 0.8740387 & 0.05560 \\
\hline Recursos humanos & 3.858514 & 1.0511388 & 0.9629435 & 0.02268 \\
\hline Dirección & 3.779555 & .07207434 & 0.8115343 & 0.07292 \\
\hline Gestión de ventas & 3.479437 & 0.9720723 & 0.8650250 & 0.08415 \\
\hline Innovación & 3.194264 & 1.0028455 & 0.8933546 & 0.19514 \\
\hline Producción-operación & 4.351293 & 0.6793136 & 0.8745174 & -0.02461 \\
\hline Mercadotecnia & 3.386117 & 0.9133799 & 0.8267290 & 0.16884 \\
\hline Finanzas & 3.802843 & 0.7583202 & 0.8809515 & 0.05766 \\
\hline Satisfacción con la empresa & 3.846652 & 0.6140355 & 0.6682914 & 0.09641 \\
\hline Ventajas competitivas & 4.149462 & 0.7309623 & 0.8822298 & -0.03201 \\
\hline Ámbitos de ventas & 3.375718 & 0.9121474 & 0.8039964 & 0.15986 \\
\hline Responsabilidad social & 3.768588 & 1.0985574 & 0.9450125 & -0.03369 \\
\hline Valoración del entorno & 3.782577 & 1.0506091 & 0.8965684 & -0.02341 \\
\hline
\end{tabular}

Fuente: elaboración propia con base en los resultados de la investigación 2020.

\section{RESULTADOS}

Para valorar el efecto de la delincuencia y la corrupción en la toma de decisiones de los directores y directoras de las mypes de Teapa, se compararon las puntuaciones de cada variable del análisis sistémico en las empresas que han sido víctimas y en las que no lo han sido. Adicionalmente, se pondero mediante regresiones lineales el efecto de la percepción de que existe violencia y corrupción en el municipio antes citado y que estas afectan al negocio.

De hecho, la corrupción que debe soportar las empresas de Teapa, Tabasco es de 3.07 que se encuentra por arriba de la media nacional que es de 2.99 y además la percepción de los directores y directoras en los actos de corrupción se encuentran en los tramites de las solicitudes de contratación de los servicios públicos. En contraparte, los trámites judiciales por conflictos administrativos, civiles, laborales se consideran los de menor acto de corrupción.

Por ejemplo en la tabla 4 se puede apreciar la diferencia de medias en relación a la delincuencia, por lo que la hipótesis es pertinente y se discuten sobre las implicaciones de éstas.

Tabla 4. Diferencia de medias de las variables de la delincuencia

\begin{tabular}{|l|c|c|c|c|c|}
\hline \multicolumn{1}{|c|}{ Variable } & $\begin{array}{c}\text { Vivió } \\
\text { delincuencia }\end{array}$ & $\begin{array}{c}\text { No vivió } \\
\text { delincuencia }\end{array}$ & T & g.I & Valor p \\
\hline Recursos humanos & 3.845 & 3.862 & -0.160 & 229 & -0.540 \\
\hline Análisis de mercado & 3.797 & 3.842 & -0.540 & 206 & 0.589 \\
\hline Proveedores & 4.103 & 4.112 & -0.110 & 220 & 0.913 \\
\hline Dirección & 3.758 & 3.785 & -0.340 & 204 & 0.732 \\
\hline Finanzas & 3.748 & 3.818 & -0.830 & 190 & 0.408 \\
\hline Gestión de ventas & 3.595 & 3.433 & 1.620 & 216 & 0.107 \\
\hline
\end{tabular}




\begin{tabular}{|l|c|c|c|c|c|}
\hline Producción - Operación & 4.272 & 4.378 & -1.390 & 188 & 0.168 \\
\hline Innovación & 3.305 & 3.151 & 1.540 & 236 & 0.124 \\
\hline Mercadotecnia con la & 3.701 & 3.607 & 1.250 & 225 & 0.214 \\
\hline $\begin{array}{l}\text { Satisfacción } \\
\text { empresa }\end{array}$ & 3.823 & 3.853 & -0.460 & 207 & 0.647 \\
\hline Ventaja competitiva & 4.082 & 4.174 & -1.190 & 210 & 0.235 \\
\hline Ámbito de ventas social & 3.226 & 3.155 & 0.690 & 229 & 0.492 \\
\hline $\begin{array}{l}\text { Responsabilidad } \\
\text { ISO }\end{array}$ & 3.899 & 3.727 & 1.760 & 258 & 0.079 \\
\hline Valoración del entorno & 3.941 & 3.983 & -0.500 & 173 & \\
\hline
\end{tabular}

Fuente: elaboración propia con base en los resultados de la investigación 2020

De igual forma, en la tabla 5 se observa que la percepción de que la delincuencia tiene un efecto en más variables y que el simple hecho del reconocimiento de su existencia sea muy parecido al de haber sido víctima, dando pie para corroborar que la idea de ser afectados por la delincuencia en sus negocios está llevando a las empresas de Teapa ajustar sus estrategias de gestión en la toma de decisiones.

Tabla 5 Percepción de la delincuencia

\begin{tabular}{|l|c|c|c|}
\hline \multicolumn{1}{|c|}{ Variable } & Existe & Afecta a mi negocio & $\begin{array}{c}\text { Veces que ha sido } \\
\text { victima }\end{array}$ \\
\hline Recursos humanos & 0.223 & .248 & -0.014 \\
\hline Análisis de mercado & 0.026 & -0.013 & -0.102 \\
\hline Proveedores & 0.127 & 0.289 & -0.075 \\
\hline Dirección & -0.024 & $-0.111-$ & -0.034 \\
\hline Finanzas & 0.149 & -0.308 & 0.015 \\
\hline Gestión de ventas & -0.106 & 0.469. & 0.038 \\
\hline Producción - operación & $0.318^{*}$ & -0.111 & -0.122. \\
\hline
\end{tabular}

Fuente: elaboración propia con base en los resultados de la investigación 2020

Por último, se presentan resultados del análisis sistémico por cada variable, en donde se ve la diferencia de medias de las empresas que mencionaron no haber participado en algún acto de corrupción versus las que si participaron tabla 6. 
Tabla 06. Impacto de la corrupción en el análisis sistémico.

\begin{tabular}{|c|c|c|c|}
\hline Variable & Descripción & $\begin{array}{l}\text { No ha participado en } \\
\text { actos de corrupción }\end{array}$ & $\begin{array}{l}\text { Participó en algún } \\
\text { acto de corrupción }\end{array}$ \\
\hline \multirow[t]{3}{*}{ Insumo del sistema } & Proveedores & 4.09 & 4.09 \\
\hline & Análisis de mercado & 3.89 & 3.79 \\
\hline & Recursos humanos & 3.89 & 3.79 \\
\hline \multirow[t]{6}{*}{ Proceso del sistema } & Dirección & 3.79 & 3.70 \\
\hline & Gestión de vtas. & 3.5 & 3.5 \\
\hline & Innovación & 3.29 & 3.29 \\
\hline & Producción -operación & 4.40 & 4.29 \\
\hline & Mercadotecnia & 3.70 & 3.60 \\
\hline & Finanzas & 3.79 & 3.79 \\
\hline \multirow[t]{5}{*}{$\begin{array}{l}\text { Resultados de } \\
\text { sistema }\end{array}$} & $\begin{array}{c}\text { Satisfacción con la } \\
\text { empresa }\end{array}$ & 3.89 & 3.79 \\
\hline & Ventaja competitiva & $4-20$ & 4.09 \\
\hline & Ámbito de ventas & 3.79 & 3.70 \\
\hline & Responsabilidad social & 3.79 & 3.70 \\
\hline & Valoración del entorno & 4 & 3.89 \\
\hline
\end{tabular}

\section{DISCUSIÓN}

Fuente: elaboración propia con base en los resultados de la investigación 2020

Los niveles de percepción de la corrupción en México son alarmantes y los intentos para reducirlos han sido un fracaso. En el 2015, México obtuvo una calificación de 35 puntos de 100 posibles y el lugar número 95 de 168 países según Transparencia Internacional (2016). Dichos datos coinciden con los del Banco Mundial, organismo que reprueba a México con una calificación de 26 (sobre 100) en sus indicadores de control de la corrupción y lo coloca en el lugar 153, es decir, como uno de los países más corruptos World Bank (2014). Si dividiéramos a los países por difíciles, México estaría en el último de ellos, al lado de países que poco o nada tienen que ver con el tamaño de nuestra economía, nivel de desarrollo o fortaleza institucional. La situación no es alentadora para México ni cuando se le compara con países miembros de organismos internacionales a los que pertenece ni tampoco cuando se le compara con países de características similares.

No obstante, El impacto económico de la violencia en México se estimó en 4.57 billones de pesos (US\$238 mil millones) en 2019, lo que equivale a $21.3 \%$ del PIB del país. Esto constituye una disminución de $0.3 \%$ en comparación con el año anterior y es la primera reducción en cinco años. Con todo, desde 2015, el impacto económico se ha incrementado $39.9 \%$, lo que refleja el deterioro de la paz (Índice de Paz México, 2020b).

Sin duda, diferentes organismos nacionales e internacionales y autores afirman que la corrupción inhibe el desarrollo económico de la empresas (Aidis \& Adachi. 2007b).

Transparencia Internacional (TI,2018) citado por Palestina (2018) el fenómeno de la corrupción 
es "La distorsión de un bien público a fines privados"; aunque acepta que el termino definido de tal manera puede resultar ambiguo en particular, si se piensa en corrupción en entidades privadas y públicas, ya que su objeto es distinto (la corrupción de entidades privadas se parece más a transacciones ilegales).

Anokhin y Schulze (2009) mencionan que la corrupción es uno de los principales obstáculos al crecimiento económico. Conforme a los resultado se concluye que, a pesar de la corrupción, las mypes del municipio de Teapa, Tabasco que más crecen, son aquellas que no han participado en actos corruptivos, aunque el crecimiento sea lento en comparación con aquellas que han participado, de la misma forma las mypes de este municipio perciben el $2.99 \%$ como actos de corrupción al realizar un trámite o cuando son objeto de supervisión en el entorno del negocio y por ende da como resultado una afectación directa en el desarrollo de estrategias operativas del mismo.

De acuerdo con Loureiro (2013), la persistencia de la delincuencia obedece al efecto histéresis presente en el comportamiento criminal. Existen dos fuentes de histéresis: la externa y la interna. La primera es aquella que viene del estigma social que provoca que los delincuentes no encuentren empleo fácilmente o que reciban un salario más bajo que el que recibirían si no fueran criminales, lo cual hace que el capital social de estos individuos se deprecie a una mayor velocidad. Este estigma social en el mercado laboral, por lo tanto, propicia que los delincuentes continúen cometiendo delitos. La fuente interna, por otro lado, proviene de la pérdida del costo moral en el momento de cometer el delito y del aprendizaje de la "tecnología" criminal; estos dos elementos interactúan en el proceso de decidir cometer un delito o no, lo cual hace que la decisión sea cada vez menos difícil.

Es importante señalar que la delincuencia es una de las variables que interfiere en la toma de decisiones dentro de las empresas estas son más propensas a percibir este fenómeno, además es uno de los problemas principales en el crecimiento de las mismas, ya que genera cambios en cuanto al comportamiento de la sociedad y además y aunado a esto la percepción de inseguridad y delincuencia por los directores y directoras de las mypes es de $15.17 \%$ resultados obtenidos de la investigación, los cuales afectan las estrategias de gestión de operatividad de su entorno empresarial, si bien los datos proporcionan información interesante que permitirán implementar planes de acción que favorezcan a esta problemática y se pueda generar otras investigaciones a corto y largo plazo.

\section{REFERENCIAS}

1. Aidis \& Adachi. Y, (2007). Sussia: Firm entry and survival barriens. Economic Systems, 31(4),391-411. https:// doi.org/10.1016/j.ecosys.2007.08.003.

2. Álvarez, C., y Urbano, D. (2011). Factores del entorno y actividad emprendedora en América Latina. Academia Revista Latinoamericana de Administración, 16760(48), 31-45.

3. Anokhin and Schulze. (2009). Entrepreneurship, innovation, and corruption. Journal of Business Venturing, 24 (2009), pp. 465-476 http://dx.doi.org/10.1016/j.jbusvent.2008.06.001.

4. Bailey, John, y Pablo, Parás. (2006). "Perceptions and attitudes about corruption and democracy in Mexico" Mexican Studies, 22(1): 57-82. https://doi.org/ 10.1525/msem.2006.22.1.57.

5. Baumol, W. J. (1990). Entrepreneurship: Productive, unproductive, and destructive. Journal of Political Economy, 98(5), 893-921. https://doi.org/10.1086/261712.

6. Bernal, M. y Castillo, R. (2012). "Efecto de la Delincuencia sobre la Inversión Extranjera Directa en México". Comercio Exterior, 62(3), 18-27.

7. Boudreaux, C. J., Nikolaev, B. N., y Holcombe, R. G. (2018). Corruption and destructive entrepreneurship. Small Business Economics, 51(1), 181-202. https://doi.org/10.1007/s11187-017-9927-x.

8. Briceño-León, R. \& Zubillaga, V. (2002). Violence and globalization in Latin America. Current Sociology, 50(1), 19-37. Recuperado de https://doi.org/10.1177/0011392102050001003.

9. Centro de investigaciones sociológicas (CIS). (2000). Dirección General de la Guardia Civil, Seguridad 
ciudadana y victimización, Estudio 2284, Boletín $\mathrm{n}^{\circ} 24$, abril-junio, disponible en www.cis.es.

10. CONEVAL. (2018). Consejo Nacional de Evaluación de la Política de Desarrollo Social. Índice de la tendencia laboral de la pobreza.

11. Corbacho, A., Philipp, J. \& Ruiz-Vega, M. (2015). Crime and Erosion of Trust: Evidence for Latin America. World Development, 70, 400-415. https://doi.org/10.1016/j.worlddev.2014.04.013.

12. Dammert, L. \& Malone, M. F. T. (2006). Does It take a Village? Policing Strategies and Fear of Crime in Latin America. Latin American Politics and Society, 48(4), 27-51.

13. Del Castillo, Arturo. (2003) Medición de la corrupción: Un indicador de la rendición de cuentas. Ciudad de México: Auditoría Superior de la Federación (Serie: Cultura de la Rendición de Cuentas, 5).

14. Del Castillo, Arturo, \& Manuel, Guerrero. (2003). Percepciones de la corrupción en la Ciudad de México. ¿Predisposición al acto corrupto? Documento de trabajo 134. Ciudad de México: CIDE.

15. DENUE. (2019). Directorio Estadístico Nacional de Unidades Económicas. México: INEGI.

16. Dimant, E., y Tosato, G. (2018). Causes and effects of corruption: what has past decade's empirical research taught us? A survey. Journal of Economic Surveys, 32(2), 335-356. https://doi.org/10.1111/joes.12198.

17. Dutta, N., y Sobel, R. (2016). Does corruption ever help entrepreneurship? Small Business Economics, 47(1), 179-199. https://doi.org/10.1007/s11187-016-9728-7.

18. ENVE. (2016).Instituto Nacional de Estadística y Geografía (INEGI). Encuesta Nacional de Victimización de Empresas. https://www.inegi.org.mx/contenidos/programas/enve/2016/doc/enve_2016_presentacion_ ejecutiva.pdf

19. ENVE. (2018).Instituto Nacional de Estadística y Geografía (INEGI). Encuesta Nacional de Victimización de Empresas. https://www.inegi.org.mx/contenidos/programas/enve/2018/doc/enve_2018_presentacion_ ejecutiva.pdf.

20. Foro Económico Mundial sobre América Latina.(2018). https://www.gob.mx/se/articulos/foro-economicomundial-sobre-america-latina-2018.

21. Friedrich, Carl (2002) "Corruption concepts in historical perspective" en Heidenheimery, Arnold y Michael Johnston (eds.) Political Corruption Concepts \& Contexts. Nueva Jersey: Transaction Publishers, pp. 1523, from Mobile Money in Afghanistan. Working paper.

22. Gardiner, John. (2002). "Defining corruption" en Heidenheimery, Arnold y Michael Johnston (eds.) Political Corruption Concepts \& Contexts. Nueva Jersey: Transaction Publishers, pp. 25-39.

23. IIEG. (2018). Instituto de Información Estadística y Geográfica con base en datos proporcionados por el Sistema de Información Empresarial Mexicano (SIEM).

24. Índice de Paz México. (2020). Identificar y medir los factores que impulsan la paz, Sídney, abril de 2020. Disponible en: indicedepazmexico.org.

25. INEGI. (2021). Encuesta Nacional de Seguridad Pública Urbana, ttps://www.inegi.org.mx/contenidos/ programas/ensu/doc/ensu2021_mar_presentacion_ejecutiva.pdf.

26. Institute for Economics \& Peace. (2017). Identificar y medir los Factores que impulsan la paz. Institute for Economics \& Peace. Global Peace Index 2018: Measuring Peace in a Complex World, Sydney, June 2018. Available from: http://visionofhumanity.org/reports (accessed Date Month Year).

27. Islam, A. (2014). Economic growth and crime against small and medium sized enterprises in developing economies. Small Business Economics, 43(3), 677-695. https://doi.org/10.1007/s11187-014-9548-6.

28. Jaitman, L. \& Keefer, P. (2017). ¿Por qué es importante la estimación de los costos del crimen? Una agenda de investigación para apoyar las políticas de prevención del delito en la región. En L. Jaitman (ed.), Los costos del crimen y de la violencia: Nueva evidencia y hallazgos en América Latina y el Caribe (pp. 1-18). Washington, D.C: Banco Interamericano de Desarrollo

29. KPC(2019).Una mirada al tema y fraudes en las empresas de América Latina. https://home.kpmg/cr/es/home/ tendencias/2020/11/corrupcion-y-fraude-en-america-latina.html.

30. KPGM.(2014).Perspectivas de la Alta Dirección en México. https:/home.kpmg/mx/es/home/sala-de-prensa/ press-releases/2014/03/cp-kpmg-presenta-perspectivas-alta-direccion-mexico-2014.html.

31. Lora, E. (2008). Calidad de vida: más allá de los hechos. Banco Interamericano de Desarrollo. https:// publications.iadb.org/publications/spanish/document/

32. Calidad-de-vida-M\%C3\%A1s-all\%C3\%A1-de-los-hechos.pdf.

33. Loureiro, André. (2013). "Essays on crime, hysteresis, poverty and conditional cash transfers", tesis de doctorado, Edimburgo, The University of Edinburgh.

34. Medina, J. (2003). Inseguridad ciudadana, miedo al delito y policía en España. Revista Electrónica de Ciencia Penal y Criminología. Recuperado de http://criminet.ugr.es/recpc/05/recpc05-03.pd.

35. Mendoza, R. U., Lim, R. A., y Lopez, A. O. (2015). Grease or Sand in the Wheels of Com-merce? Firm Level Evidence on Corruption and SMES. Journal of International Develo-pment, 27(4), 415-439. https://doi. org/10.1002/jid.3077.

36. Mertz, C. (2012). Delincuencia en Chile: diagnóstico y propuestas. Estudio de Trasfondo. 95 propuestas para 
un Chile mejor. Santiago de Chile.

37. Morlai, A. (2015). The effect of corruption on small and medium entreprises: Perspective from a developing country. International Journal of Small Business and Entrepreneurs-hip Research, 3(3), 12-27.

38. Motta, V. (2017). The impact of crime on the performance of small and medium-sized enterprises: Evidence from the service and hospitality sectors in Latin America. Tourism Economics, 23(5), 993-1010.

39. Mthimkhulu, A. M. \& Aziakpono, M. J. (2018). What impedes micro, small and medium firms' growth the most in South Africa? Evidence from World Bank Enterprise Surveys. South African Journal of Business Management, 46(2), 15-27.

40. Naval, Claire, y Juan, Salgado. (2006). Irregularidades, abusos de poder y maltrato en el Distrito Federal. La relación de los agentes policiales y del Ministerio Público con la población: Fundar.

41. Palestina,I.E.(2018).Corrupción y crecimiento económico: las percepciones de la sociedad colombiana. Revista CES Derecho, (9), 1, enero - junio 2018, 59-72.

42. Quiroz, J., Castillo, R., Ocegueda, J., y Varela, R. (2015). Delincuencia y actividad económica en México. Norteamérica, 10 (2).

43. Ramírez, S. R. (2019). Del Edén al infierno: inseguridad y construcción Estatal en Tabasco. DOI: http://dx.doi. org/10.29043/liminar.v17i2.690.

44. Rodriguez, J. J. (12 de Octubre de 2017). Robo a negocio: cifra más alta de los últimos 21 años. Recuperado el 30 de Noviembre de 2017, de El Universal: http://www.eluniversal.com.mx/observatorio-nacional-ciudadano/ robo- negocio-cifra-mas-alta-de-los-ultimos-21-años.

45. Ruidíaz, C. (2003). "El miedo al delito. Apuntes para la reflexión", Cuadernos de Política criminal, nº 48, págs. 931 ss.

46. Sabet, Daniel. (2013). "Corruption or insecurity? Understanding dissatisfaction with Mexico's police" Latin American Politics and Society, 55(1): 22-45I, DOI: https://doi.org/10.1111/j.1548-2456.2012.00182.x

47. Sansó-Rubert, Daniel. (2005). La internacionalización de la delincuencia organizada: análisis del fenómeno UNISCI Discussion papers Seminario de Estudios de Defensa, Universidad de Santiago de Compostela Núm. 9.

48. Secretaría de Economía. (2019). Microempresas. Agosto 30,2019, de economia.gob.mx Sitio web: http:// www.2006-012.economia.gob.mx/mexicoemprende/empresas/microempresario.

49. Soares, R. R. \& Naritomi, J. (2010). Understanding High Crime Rates in Latin America. En R. Di Tella, S. Edwards \& E. Schargrodsky (eds.), The Economics of Crime (pp. 19-55).

50. Soria-Romo, Rigoberto. (2017). Impacto de la violencia e inseguridad en la competitividad de los estados mexicanos, Economía, Sociedad y Territorio, vol. XVII, núm. 54, mayo-agosto, 2017, pp. 279-307, El Colegio Mexiquense, A.C.Toluca, México.

51. Soto, S., (2005). La influencia de los medios en la percepción social de la delincuencia. Revista Electrónica de Ciencia Penal y Criminología. núm. 07-09, p. 09:1-09:46. Tourism Economics, 23(5), 993-1010.

52. Transparency International Secretariat. (2018). Corruption Perceptions Index 2017 shows high corruption burden in more than two-thirds of countries. Recuperado de https://www.transparency.org/news/pressrelease/ el índice de percepción de la corrupción 2017 muestra una fuerte presencia.

53. Transparency international. (2017). Índice de Percepción de corrupción, disponible en: http://www. transparency.org/news/feature/corruption perceptions index2016 (Fecha de consulta. 10 de octubre del 2018).

54. Transparencia Internacional. (2017). What is Corruption? [en línea]. Disponible en: <https://www.transparency. org/what-is-corruption/>.

55. Transparency International. Anti-Corruption Glosary.(2016). https://www.transparency.org/glossary/. University of Chicago Press. https://doi.org/10.7208/chicago/9780226153766.003.0002.

56. UNODC. (2019). Visible: Homicide Dataset 2019. National Data. Recuperado de https://dataunodc.un.org/ GSH_app

57. Vilalta, C. (2016). La victimización de las empresas en México: conceptos, teorías y mediciones. https://onc. org.mx/uploads/CompetitividadEmpresarial.pdf.

58. Williams, N., y Vorley, T. (2015). Institutional asymmetry: How formal and informal institutions affect entrepreneurship in Bulgaria. International Small Business Jour-nal: Researching Entrepreneurship, 33(8), 840-861. https://doi.org/10.1177/0266242614534280.

59. World Bank.(2014). Worldwide Governance Indicators, http://info.worldbank.org/governance/wgi/index. aspx\#home. 